\title{
Prevalence of high blood pressure among schoolchildren from Córdoba, Argentina, and its relation to socioeconomic status
}

\author{
Agustin González Calbano, M.D. ${ }^{a}$, Manuel Álvarez Moyano, M.D. ${ }^{b}$ \\ Prof. Verónica Mamondi, MPH and Prof. Silvina Berra, PhD. ${ }^{d}$
}

a. Department of Health Sciences, Universidad Nacional del Sur, Bahía Blanca.

b. Department of Clinical Cardiology and Cardiothoracic Surgery, Hospital Córdoba.

c. School of Nutrition, Facultad de Ciencias Médicas, Universidad Nacional de Córdoba.

d. Center for Research and Studies on Culture and Society (CIECS), National Scientific and Technical Research Council (CONICET), Universidad Nacional de Córdoba, Argentina.

E-mail address: Silvina Berra, PhD: sberra@fcm.unc.edu.ar

\section{Funding:}

Research and Development Project 2010. Res. no. $000153 / 2011$. Ministry of Science and Technology of the province of Córdoba.

Conflict of interest: None.

Received: 11-2-2017 Accepted: 4-19-2018

\section{ABSTRACT}

Introduction. Prior studies conducted in Argentina found variable prevalence rates of high blood pressure(HBP) among children using different measurement methods. The objective of this study was to know the prevalence of HBP among schoolchildren from the city of Córdoba and its relation to sex, nutritional status, socioeconomic status (SES), and level of maternal education.

Population and methods. Blood pressure measurements and information on SES, level of maternal education, and body mass index of schoolchildren from the city of Córdoba, Argentina, were obtained. HBP was defined as systolic or diastolic blood pressure levels that are greater than or equal to the 95th percentile for a given age, sex, and height percentile at three consecutive measurements on separate days. Results. A total of 1531 boys and girls participated; their average age was 10.6 years. Fifty-five children had HBP. Systolic and diastolic blood pressure levels showed a significant association with obesity $(\mathrm{p}=0.001)$, but not with age $(\mathrm{p}=0.87)$, sex (systolic: $\mathrm{p}=0.48$; diastolic: $\mathrm{p}=$ 0.71 ) or SES (systolic: $\mathrm{p}=0.07$; diastolic: $\mathrm{p}=0.09$ ). Conclusions. The prevalence of HBP in a sample of schoolchildren from Argentina was 3.7\%. It showed a significant association with obesity, but not with SES.

Key words: hypertension, child, socioeconomic factors, body mass index.

http: / / dx.doi.org/10.5546/ aap.2018.eng.340

To cite: González Calbano A, Álvarez Moyano M, Mamondi V, Berra S. Prevalence of high blood pressure among schoolchildren from Córdoba, Argentina, and its relation to socioeconomic status. Arch Argent Pediatr 2018;116(5):340-344.

\section{INTRODUCTION}

Traditionally, high blood pressure (HBP) used to be diagnosed in adult life; however, the evidence of vascular damage developing in the first years of life has prompted its study at earlier ages. In addition to the different risk factors that have been associated with essential HBP among children, specifically obesity, studies have suggested that social determinants such as socioeconomic status (SES) and the level of maternal education may also be associated with this diagnosis. ${ }^{1,2}$

It is known that pediatric blood pressure (BP) varies and a child's $B P$ readings may fluctuate day by day or even within the same day. ${ }^{3}$ This may be due to the "alerting response", which is defined as an increase in BP noticed during the medical consultation. Because of this phenomenon, $30-50 \%$ of children with abnormal BP values at the physician's office have normal values once outside. ${ }^{4,5}$ Such effect is known as "white coat hypertension" and there is no consensus on its impact or management. ${ }^{6}$ In this situation, ambulatory blood pressure monitoring (ABPM) over 24 hours defines the presence or absence of white coat hypertension. Likewise, there is a reverse effect known as "masked hypertension," that refers to the presence of normal measurements at the physician's office and abnormal ambulatory values. ${ }^{7}$

However, ABPM is complicated and costly, and requires staff especially trained on this method. ${ }^{8}$ For this reason, the National High Blood Pressure Education Program Working Group on Children and Adolescents of the National Heart, Lung and Blood Institute (NHLBI) of the United States has recommended the use of three repeated ambulatory measurements on separate occasions, far from each other. $^{8}$

In Argentina, prior studies found that the prevalence of HBP ranged from $1.4 \%$ to $13.4 \%,{ }^{9}$ although only the studies by Dumas et al. and Díaz et al. used the NHLBI's criterion. ${ }^{10,11}$ 
The variability in the prevalence observed by the different studies may be due to the use of diverse methods to measure $\mathrm{BP}$, as shown by the systematic reviews done in Brazilian children and adolescents. ${ }^{12,13}$ Likewise, among Argentine children, there is a known association between HBP and typical risk factors, but there is no knowledge on its relation to SES, which is especially relevant in countries with a highly unequal income distribution, as observed in Latin America.

The objective of this study was to know the prevalence of HBP among schoolchildren from the city of Córdoba and its relation to sex, nutritional status, SES, and level of maternal education.

\section{POPULATION AND METHODS}

The study population was made up of regular students who were in fourth, fifth, and sixth grade in 2011 at the 37 municipal public schools from the city of Córdoba (Argentina), which, in March 2011, had 4900 children enrolled. Children represented the study's unit of analysis and schools, the unit of sampling. Two school groups were established: the first one included those where $25 \%$ or less of parents had no education or incomplete primary education ( $n=20$ schools) and the second one was made up of those schools where such level of education corresponded to more than $25 \%$ of parents ( $\mathrm{n}=17$ schools). Nineteen schools were randomly SESected (10 from the first group and 9 from the second one) and all students from fourth to sixth grade were included, for a total of 2423 schoolchildren. The following exclusion criteria were applied: children who refused to have measurements taken, children whose parents or legal guardians refused to give their consent to participate, children who were absent at the time of study measurements, and children with known secondary HBP.

BP was measured using an oscillometric sphygmomanometer. The oscillometric method analyzes pulse waves instead of the audible blood flow; therefore, although using an automatic device rules out observer bias, each manufacturer uses its own algorithms to obtain BP measurements, and this may lead to variable readings among the different devices..$^{14}$ In order to minimize such differences, we used the OMRON 705CP-II oscillometric sphygmomanometer, which has been validated for its use in children and adolescents. ${ }^{15}$

Measurements were taken in a quiet room at the right temperature, with the child sitting down after resting for 3-5 minutes. The cuff was positioned on the right arm, at the height of the heart, according to the recommendations made by the American Heart Association and the NHLBI. ${ }^{8}$ A single measurement was taken per visit. Children with elevated BP for their age and height at the first measurement were reassessed at a second visit. Likewise, children with elevated $\mathrm{BP}$ at the second visit were reassessed at a third, final visit. Since it was not possible to reassess all children who had been asked to return for a second and third visit, analyses were done considering the initial number of assessed children.

Height was measured using a portable stadiometer with a $0.1 \mathrm{~cm}$ precision and a range up to $200 \mathrm{~cm}$, attached to a wall and with a rigid platform placed in the base. Children were measured in light clothing and barefoot, with their back against the scale of the stadiometer. Height was classified in percentiles according to the national reference standards. ${ }^{17} \mathrm{BP}$ percentiles were estimated according to the NHLBI standards. ${ }^{8}$ Elevated BP was defined as systolic blood pressure (SBP) or diastolic blood pressure (DBP) greater than or equal to the $95^{\text {th }}$ percentile (P95) for a given age, sex, and height percentile. HBP was defined as a BP greater than or equal to the P95 for age, sex, and height percentile in three measurements on separate nonconsecutive days.

Data on age (years), sex, level of maternal education, and SES were also collected. The SES was measured using the Family Affluence Scale (FAS), which assesses the child's household material resources in terms of number of cars and computers owned by the family, whether the child has his/her own room, and family vacations in the past 12 months. The questionnaire was completed by children themSESves in the classroom, after receiving an explanation by a trained member of the research team.

Before developing field activities, a pilot test was carried out in one of the schools in order to test the study feasibility and make the necessary adjustments to minimize errors during data collection. This was also helpful to train the team on the study protocol regarding anthropometric and blood pressure measurements in the pediatric setting to reduce intra- and inter-observer variability.

Data were analyzed using the SPSS software, version 18 for Windows. The descriptive statistics of the sample characteristics included measures of central tendency and dispersion for quantitative 
outcome measures and frequency distribution for categorical outcome measures. The association among outcome measures was assessed using the $\chi^{2}$ test for categorical outcome measures and Student's $t$ test or an analysis of variance (ANOVA) for quantitative outcome measures once it was confirmed that data had a normal distribution.

The protocol was reviewed and approved by the Institutional Ethics Committee in Health Research (Comité Institucional de Ética en Investigación en Salud, CIEIS) of Hospital Nacional de Clínicas de Córdoba (file no. 066/10). The results of children with confirmed HBP were communicated to their parents, together with a recommendation to see a pediatrician.

\section{RESULTS}

A total of 1531 children participated out of 2423 who had been SESected. The following are the reasons why some children did not take part in the study: 748 did not have their parents' or legal guardian's informed consent, 98 were absent at the time of the measurements, and 46 refused to participate on their own. Participants' average age was 10.6 years (range: $8-14$ ), and no significant differences were observed by sex $(p=0.95)$. At the time of the survey, 6 children in fourth grade had not turned 9 years old yet.

Also, $54 \%$ of participants were girls; $21.4 \%$ had a low SES; $72.4 \%$ of mothers had completed primary education or less; $15.2 \%$ of children were obese, and $22.7 \%$, overweight. At the first measurement, $24 \%$ of children had a $\mathrm{BP} \geq \mathrm{P} 95$; at the second measurement, $7.5 \%$; and at the final measurement, 3.7\% (55 children, 95\% confidence interval [CI]: 2.9-4.8), based on the criterion of three measurements greater than or equal to the P95 on nonconsecutive days (Table 1). At the second measurement, 55 children refused to participate or were absent $(3.7 \%)$.

The prevalence of HBP was higher in the group of obese children $(\mathrm{p}<0.01)$. The prevalence in the 8-to-10-year-old group was $3.4 \%$, whereas in the 11-to-14- year-old group, it was $4.1 \%$. Mean SBP and DBP were higher in the 11-to-14-yearold group, but no significant differences were observed (Table 2). Also, no significant difference was noted in terms of sex, age, SES, and level of maternal education (Table 2).

\section{DISCUSSION}

Findings are consistent with those of Dumas et al. and Díaz et al., who found, using a similar methodology, a prevalence of 3.4\% (95\% CI: 2.44.7 ) and $4.3 \%$ (95\% CI: 2.3-7), respectively. ${ }^{10,11}$ In a sample of younger children, Szer et al. found a significantly higher prevalence (95\% CI: 7.4-11.6), but, unlike Dumas et al., that study made three BP measurements within the same day..$^{18}$ Other studies were done in older children or using different methodologies. ${ }^{9}$

At a regional level, two studies using a similar methodology found mixed results: in Brazil, Rinaldi et al. found a prevalence of $3.2 \%$, whereas Ferreira et al. reported a prevalence of $1.9 \% .{ }^{19,20}$ Also in Brazil, Nogueira et al. used only two measurements but with a 1-year interval and described a prevalence of $0.6 \% .{ }^{21}$ The two latter results are significantly different from our results. At an international level, in the United States and using repeated measurements on separate occasions, Sorof et al. found a $4.5 \%$ prevalence (95\% CI: $4-5.1)$ and Hansen et al. described a $3.6 \%$ prevalence (95\% CI: 3.3-3.9). ${ }^{16,22}$ None of those studies showed significant differences with our study.

It is worth noting that HBP prevalence at the first measurement was $24 \%$, significantly higher than what has been observed in other studies. In addition, the mean BP value at the first measurement was also higher than that of other studies. ${ }^{19}$ This may be because we did a single measurement at the first visit and also probably because this was the first time participating

TABLE 1. Prevalence of high blood pressure at each visit, in relation to the initial number of assessed children

\begin{tabular}{|c|c|c|c|c|c|c|c|c|c|c|}
\hline & \multicolumn{2}{|c|}{ First visit } & \multicolumn{3}{|c|}{ Second visit } & \multicolumn{2}{|c|}{ Third visit } & \multicolumn{3}{|c|}{ Total } \\
\hline & $\mathbf{n}$ & $\%$ & $\mathbf{n}$ & $\%^{a}$ & $\%$ valid $^{\mathrm{b}}$ & $\mathbf{n}$ & $\%$ b & $\mathbf{N}$ & $\%^{\mathrm{b}}$ & $\%$ valid $^{\mathrm{b}}$ \\
\hline$<$ P95 & 1164 & 76.0 & 202 & 13.2 & 13.7 & 55 & 3.7 & 1421 & 92.8 & 96.3 \\
\hline$\geq$ P95 & 367 & 24.0 & 110 & 7.1 & 7.5 & 55 & 3.7 & 55 & 3.6 & 3.7 \\
\hline Lost-to-follow-up & & & 55 & 3.6 & & 0 & 0 & 55 & 3.6 & \\
\hline Total & 1531 & 100 & 367 & 24.0 & & 110 & 7.5 & 1531 & 100 & \\
\hline
\end{tabular}

a The percentage estimation includes the 1531 cases.

${ }^{\mathrm{b}}$ The valid percentage estimation excluded the 55 cases who were lost-to-follow-up at the second visit, for a total of 1476 cases. 
children had their BP measured.

The results confirm the relation between childhood obesity and HBP. Given the close relation between childhood HBP and obesity, several studies have pointed out the correlation between the increase of overweight and obesity prevalence and the increase of HBP prevalence. ${ }^{23,24}$ However, in a recent review, this correlation does not seem to be absolute and, in several countries, the increase in the prevalence of obesity showed a negative correlation to HBP prevalence; this suggests that the role of other determinants, such as low birth weight, family history, salt intake, and physical activity, among others, should be studied. ${ }^{25}$

Prior studies on the role of SES in children's BP used the level of maternal education as a SES indicator ${ }^{1}$ or questionnaires that had not been validated to assess income adequacy. ${ }^{2}$ In our study, no significant differences were observed by SES, which was measured using a validated questionnaire. The level of maternal education was not associated with BP, except for DBP. However, our sample was restricted to public schools and was not representative of the entire Córdoba population because private schools were specifically excluded.

The major limitation of this study was a suboptimal participation rate and the corresponding loss to follow-up of some participants whose BP should have been reassessed. These children often miss school; it may be assumed that such behavior is associated with more unfavorable family conditions. However, the study by Sorof et al. described a similar rate of loss to follow-up, and their HBP prevalence was not significantly different from our study or other studies using the same methodology. ${ }^{16}$

The main strength of this study was the use of the recommended methodology to define HBP in children. Other strengths were that the sample size was large enough for an adequate accuracy, data were collected by a trained team to minimize intra- and inter-observer variability, and that the SES indicator of our study is widely used at the international level.

\section{CONCLUSION}

The prevalence of HBP in a sample of schoolchildren from Argentina, based on the internationally recommended methodology, was $3.7 \%$. The prevalence of HBP in this sample showed a significant association with the prevalence of obesity, but not with SES.

TABLE 2. Systolic and diastolic blood pressure values and percentage classified as high blood pressure after three measurements on separate days in students from municipal schools of the city of Córdoba, 2011

\begin{tabular}{|c|c|c|c|c|c|c|c|}
\hline Characteristics & $\mathbf{n}$ & Average SBP & P ANOVA & Average DBP & P ANOVA & $\%$ with HBP (n) & $\mathbf{P} \chi^{2}$ \\
\hline \multicolumn{8}{|l|}{ Sex } \\
\hline Male & 675 & 110.74 & 0.482 & 64.06 & 0.717 & $4.6(31)$ & 0.07 \\
\hline Female & 801 & 110.41 & & 64.28 & & $3.0(24)$ & \\
\hline \multicolumn{8}{|l|}{ Age } \\
\hline 8 to 10 years old & 734 & 107.75 & 0.937 & 63.78 & 0.252 & $3.4(24)$ & 0.46 \\
\hline 11 to 14 years old & 797 & 113.16 & & 64.56 & & $4.1(31)$ & \\
\hline \multicolumn{8}{|l|}{ SES } \\
\hline Low & 318 & 110.06 & 0.074 & 64.03 & 0.089 & $4.2(13)$ & 0.90 \\
\hline Middle & 797 & 110.74 & & 64.21 & & $3.7(28)$ & \\
\hline High & 371 & 110.66 & & 64.44 & & $3.9(14)$ & \\
\hline \multicolumn{8}{|l|}{ Level of maternal education } \\
\hline Low (primary education or less) & 318 & 110.24 & 0.16 & 63.78 & 0.04 & $3.7(32)$ & 0.59 \\
\hline Middle (secondary education or less) & 797 & 111.35 & & 65.66 & & $4.1(11)$ & \\
\hline High (university education or less) & 371 & 110.68 & & 64.29 & & $6.4(4)$ & \\
\hline \multicolumn{8}{|l|}{ Nutritional status (WHO): } \\
\hline Underweight-normal weight & 916 & 106.28 & $<0.01$ & 62.28 & $<0.01$ & $0.7(6)$ & $<0.01$ \\
\hline Overweight & 335 & 113.86 & & 64.76 & & $3.6(12)$ & \\
\hline Obesity & 224 & 121.81 & & 70.4 & & $16.5(37)$ & \\
\hline
\end{tabular}

SBP: systolic blood pressure; DBP: diastolic blood pressure; ANOVA: analysis of variance; HBP: high blood pressure; SES: socioeconomic status; WHO: World Health Organization. 


\section{REFERENCES}

1. Bouthoorn SH, Van Lenthe FJ, De Jonge LL, et al. Maternal educational level and blood pressure, aortic stiffness, cardiovascular structure and functioning in childhood: the generation R study. Am J Hypertens 2014; 27(1):89-98.

2. Van den Berg G, van Eijsden M, Galindo-Garre F, et al. Explaining socioeconomic inequalities in childhood blood pressure and prehypertension: the $\mathrm{ABCD}$ study. Hypertension 2013; 61(1):35-41.

3. Osborne R, Mullin CS, Roberson PK. The variability of blood pressure measurements in children. Am J Public Health 1983; 73(10):1207-10.

4. Ogborn MR, Crocker JF. Investigation of pediatric hypertension. Use of a tailored protocol. Am J Dis Child 1987; 141(11):1205-9.

5. Tschumi S, Noti S, Bucher BS, et al. Is childhood blood pressure higher before or after clinic consultation? Acta Paediatr 2011; 100(5):775-7.

6. Jurko A, Minarik M, Jurko T, et al. White coat hypertension in pediatrics. Ital J Pediatr 2016; 42:4.

7. Pickering TG, Davidson K, Gerin W, et al. Masked hypertension. Hypertension 2002; 40(6):795-6.

8. National High Blood Pressure Education Program Working Group on Children and Adolescents. The Fourth Report on the Diagnosis, Evaluation, and Treatment of High Blood Pressure in Children and Adolescents. Pediatrics 2004;114(2 Suppl 4th Report):555-76.

9. Díaz A, Calandra L. Presión arterial elevada en niños y adolescentes escolarizados de Argentina en los últimos 25 años: revisión sistemática de estudios observacionales. Arch Argent Pediatr 2017; 115(1):5-11.

10. Dumas LV, López Cross SA, Peroni DS, et al. Detección de hipertensión arterial en niños en edad escolar. Rev Fac Cien Med (Córdoba) 2005; 62(3):47-52.

11. Díaz A, Tringler M, Molina JD, et al. Control de la presión arterial y prevalencia de hipertensión arterial en niños y adolescentes de una población rural de Argentina: Datos preliminares del Proyecto Vela. Arch Argent Pediatr 2010; 108(1):68-70.

12. Bezerra $M$, Soares $P$, Leite E, et al. Hipertensão em criançase adolescentes: revisão sistemática sobre prevalência e fatores de risco. Rev Enferm UFPE on line 2013; 7(8):5813-22.
13. Magalhaes MG, Oliveira LM, Christofaro DG, et al. Prevalence of high blood pressure in Brazilian adolescents and quality of the employed methodological procedures: systematic review. Rev Bras Epidemiol 2013; 16(4):849-59.

14. Bonnafoux P. Auscultatory and oscillometric methods of ambulatory blood pressure monitoring, advantages and limits: a technical point of view. Blood Press Monit 1996; 1(3):181-5.

15. Stergiou GS, Yiannes NG, Rarra VC. Validation of the Omron 705 IT oscillometric device for home blood pressure measurement in children and adolescents: the Arsakion School Study. Blood Press Monit 2006; 11(4):229-34.

16. Sorof JM, Lai D, Turner J, et al. Overweight, ethnicity, and the prevalence of hypertension in school-aged children. Pediatrics 2004; 113(3 Pt 1):475-82.

17. De Onis M, Onyango AW, Borghi E, et al. Development of a WHO growth reference for school-aged children and adolescents. Bull World Health Organ 2007; 85(9):660-7.

18. Szer G, Kovalskys I, De Gregorio MJ. Prevalencia de sobrepeso, obesidad y su relación con hipertensión arterial y centralización del tejido adiposo en escolares. Arch Argent Pediatr 2010; 108(6):492-8.

19. Rinaldi AEM, Nogueira PCK, Riyuzo M, et al. Prevalência de pressão arterial elevada em crianças e adolescentes do ensino fundamental. Rev Paul Pediatr 2012; 30(1):79-86.

20. Ferreira CEF, Faria RJ, Bazoni PS. Hipertensão arterial em crianças e adolescentes moradores da região do Caparaó, ES, Brasil. Rev Bras Cardiol 2013; 26(4):267-71.

21. Nogueira PCK, Costa RFd, Cunha JSN, etal. Pressão arterial elevada em escolares de Santos: relação com a obesidade. Rev Assoc Med Bras 2007; 53(5):426-32.

22. Hansen ML, Gunn PW, Kaelber DC. Underdiagnosis of hypertension in children and adolescents. JAMA 2007; 298(8):874-9.

23. Rosner B, Cook NR, Daniels S, et al. Childhood blood pressure trends and risk factors for high blood pressure: the NHANES experience 1988-2008. Hypertension 2013; 62(2):247-54.

24. Din-Dzietham R, Liu Y, Bielo MV, et al. High blood pressure trends in children and adolescents in national surveys, 1963 to 2002. Circulation 2007; 116(13):1488-96.

25. Roulet $C$, Bovet $P$, Brauchli $T$, et al. Secular trends in blood pressure in children: A systematic review. JClin Hypertens (Greenwich) 2017; 19(5):488-97. 